\title{
O PENSAMENTO COMPUTACIONAL NOS ANOS INICIAIS DO ENSINO FUNDAMENTAL
}

\author{
André koscianski - UTFPR - koscianski@ utfpr.edu.br \\ Fabiana Rodrigues de Oliveira Glizt - UTFPR - fabby.roliveira@ gmail.com
}

\begin{abstract}
Resumo: O raciocínio lógico está presente em atividades em todas áreas de conhecimento, mas de forma geral não é tratado como prioridade no processo de ensino. Torna-se necessário criar estratégias para que esta habilidade seja desenvolvida desde os primeiros anos de escolarização. $\mathrm{O}$ pensamento computacional (do inglês, computational thinking) engloba métodos para solução de problemas baseado nos fundamentos e técnicas da Ciência da Computação, e atualmente é visto como uma das formas de desenvolver o raciocínio lógico. Neste trabalho buscou-se, a partir da introdução de conceitos computacionais, trabalhar o processo de raciocínio dos alunos por meio de atividades lúdicas, em uma turma do ensino fundamental. Exemplos incluem conversão de números binários, métodos de ordenação, representação de algoritmos. A pesquisa pautou-se nas ideias do construtivismo de Piaget, do construcionismo de Papert, e no método desenvolvido por Polya acerca da resolução de problemas. Os resultados deste estudo fornecem evidências de que, quando os alunos têm a oportunidade de se envolver em atividades que abrangem os conceitos computacionais, habilidades além do raciocínio lógico são exercitadas e desenvolvidas, possibilitando ainda que adquiriram conhecimentos antes delimitados a áreas específicas, como computação e engenharias.
\end{abstract}

Palavras-chave: pensamento computacional; raciocínio lógico; anos iniciais.

\section{COMPUTATIONAL THINKING IN THE INITIAL YEARS OF ELEMENTARY SCHOOL}

Abstract: Logical reasoning is applied in activities in all knowledge areas, but is rarely prioritised during teaching processes. This points the need to create strategies to work with this skill since the earliest years of schooling. Computational thinking encompasses problem-solving methods based on concepts and techniques of computer science. It is currently seen as a ways to develop logical reasoning. In this work, computational concepts were introduced in activities of elementary students, in a playful manner. Examples include conversion of binary numbers, ordering methods, algorithm representation. The research was based on the ideas of Piaget's constructivism, the constructionism of Papert, and methods devised by Polya concerning problem solving. The results of this study provide evidence that when students have the opportunity to engage in activities that encompass computational concepts, they exercice and develop such skills. It also enables them to acquire knowledge ordinarily restricted to specific areas of activity, such as computing and engineering.

Keywords: computational thinking; logical reasoning; elementary school.

\section{Introdução}

Nos primeiros anos de estudo da educação básica, costuma-se delegar a uma única professora a tarefa de ensinar os conteúdos programáticos do ano em que o 
estudante está inserido. Até o terceiro ano do ensino fundamental, o aluno deverá estar plenamente alfabetizado e dominando a utilização das quatro operações básicas da matemática, para que possa utilizar em suas relações cotidianas dentro e fora do âmbito escolar.

No processo de aprendizagem dos estudantes do ensino fundamental, percebe-se que muitos apresentam dificuldades na formação do raciocínio lógico. É comum, durante a aplicação de exercícios matemáticos, alunos questionarem professores sobre, por exemplo, realizar uma operação de soma ou de subtração, sem antes procurar refletir sobre o que é proposto. Muitas vezes, o estudante que faz tal tipo de questionamento precisa de um trabalho em torno de habilidades como análise, síntese, comparação. Alguns alunos habituam-se a saltar de enunciado para a solução da atividade a partir de respostas do professor. Além disso, alunos também apresentam dificuldades na organização dos dados extraídos para realizar os exercícios.

Esse cenário dificulta o trabalho do professor e é queixa de vários profissionais em diferentes níveis de ensino. Diferente de matérias regulares no currículo escolar como português e matemática, o ensino da lógica não é considerado uma disciplina específica dos anos iniciais.

É importante destacar que, apesar dos baixos índices nacionais, este item específico - a dificuldade de desenvolver o raciocínio lógico - não é um caso particular. Dados do ano de 2014 do Programa Internacional de Avaliação de Alunos (PISA), mostram o Brasil na posição 38 entre 44 países que tiveram a habilidade de seus estudantes avaliada.

A formação do raciocínio lógico é um fator preocupante no processo de formação dos alunos; tal dificuldade pode acompanhar o estudante até o ensino superior, se não estimulada (KRAMER, 2007, pags, 36-42).

Raciocínio e lógica são palavras que se relacionam, visto que raciocinar requer a organização do pensamento de forma lógica. E é através desta organização mental ou operacional (códigos escritos e numéricos), que o estudante poderá combinar conceitos, ideias e informações para resolver problemas. Apesar de muitas vezes associar o raciocínio lógico apenas ao ensino da matemática, esta aprendizagem perpassa todas as áreas do conhecimento humano e, assim, pode ser trabalhada em várias disciplinas.

$\mathrm{O}$ pensamento computacional (do inglês, computational thinking) engloba métodos para solução de problemas baseado nos fundamentos e técnicas da Ciência da Computação, e é visto como uma das formas de desenvolver o raciocínio lógico. Neste sentido, por meio do desenvolvimento de tais fundamentos, o aluno poderá desenvolver técnicas como abstração, organização e execução passo a passo para resolução de problemas, o que irá auxiliá-lo na elaboração do seu pensamento (KRAMER, 2007, pags, 36-42). Além disso, a partir de tais conceitos, o estudante deixará de compreender o computador como mero artefato tecnológico, mas como um potencializador de suas atividades.

Para que o processo de inserção e utilização da tecnologia no espaço educativo seja realizado de forma a desenvolver habilidades cognitivas nos alunos, é fundamental que objetivos corretos sejam estabelecidos: integrar computadores em salas de aula muitas vezes esbarra em pressões da comunidade, imprensa e outros setores, sem que se embase teoricamente resultados concretos e métodos para atingi-los. O letramento computacional torna-se uma realidade em muitas escolas brasileiras, mas o trabalho acerca do pensamento computacional é recente e diz respeito a fatores mais complexos do que manipular um computador ou celular.

Considerando os dados apresentados e as observações evidenciadas no processo de aprendizagem de estudantes, o objetivo desta pesquisa é analisar as 
contribuições do pensamento computacional no desenvolvimento do raciocínio lógico dos alunos nos primeiros anos de escolarização.

Uma vez que o raciocínio lógico é uma habilidade indispensável para os estudantes, nos diferentes níveis de ensino, as aprendizagens e avanços conquistados a partir da introdução do pensamento computacional contribuirão nas diferentes disciplinas do currículo escolar.

\section{O DESENVOLVIMENTO DO PENSAMENTO INFANTIL}

Para compreender as fases do desenvolvimento biológico e pensamento, um dos pilares do projeto foram os estudos realizados por Jean Piaget. Essa referência ajuda a pautar encaminhamentos adotados com estudantes de baixa idade. Com relação à introdução de conceitos relacionados à Ciência da Computação dentro do trabalho de desenvolvimento do raciocínio lógico dos estudantes, buscaram-se as contribuições do construcionismo de Seymour Papert, que ao longo de sua vida de pesquisa se debruçou sobre a ideia de usar a tecnologia como ferramenta mediadora na produção do conhecimento. Finalmente, os estudos de Polya acerca de métodos de resolução de problemas possibilitaram a compreensão e o aprimoramento no processo de raciocínio dos alunos; eles apontam condicionantes para que o indivíduo seja capaz de encontrar soluções a partir de experiências e situações a ele apresentadas.

\section{PROCESSOS DE APRENDIZAGEM E A INFORMÁTICA EDUCATIVA: CONTRIBUIÇÕES DE JEAN PIAGET E SEYMOUR PAPERT}

Ao considerar a lógica como ciência que estuda as ideias e os processos da mente humana, compreende-se sua importância na construção de conhecimento (EVANS, 2003, pags. 454-459). Logo, é importante que seja estimulada desde os primeiros anos de estudos dos alunos.

Piaget, psicólogo e biólogo desenvolveu pesquisas acerca do desenvolvimento biológico e os processos de formação do raciocínio infantil. Apesar de ter estudado este campo, curiosamente ele não desenvolveu uma "teoria pedagógica"; no entanto, suas contribuições auxiliam e fazem parte da compreensão do conhecimento e das habilidades cognitivas dos estudantes.

Piaget buscou mostrar que o conhecimento evolui progressivamente através de estruturas de raciocínio que substituem umas às outras por meio de estágios de desenvolvimento. A teoria denominada epistemologia genética, foi definida pelo próprio Piaget (1974, p.20) como o "estudo da passagem dos estados inferiores do conhecimento aos estados mais complexos ou rigorosos". Essa teoria baseou-se em observações do desenvolvimento natural infantil, dividindo tal processo em quatro estados: sensório-motor ( 0 a 2 anos); pré-operátorio (2 a 6 anos); operátório-concreto (7 a 11 anos) e por fim o operatório-formal (12 anos em diante). Esse processo perpassa desde o nascimento da criança até sua adolescência, quando a capacidade plena do raciocínio seria atingida.

Os apontamentos da teoria de Piaget são muitos significativos para que professores possam entender os estudantes e assim selecionar e preparar atividades adequadas.

Tendo em vista a introdução de conceitos da Ciência da Computação no âmbito escolar, apontada por estudos recentes como um facilitador no desenvolvimento do raciocínio lógico dos estudantes, buscou-se contribuições do matemático e educador Seymour Papert. Papert introduziu a tecnologia como ferramenta modificadora de 
relações de aprendizagem. Ele demonstrou parte de suas ideias na prática, elaborando uma linguagem de programação de fácil compreensão, denominada LOGO, que teve repercussão mundial.

Seguidor e aluno de Jean Piaget, Papert desenvolveu o conceito do construcionismo. O Construcionismo é uma reconstrução teórica a partir dos estudos desenvolvidos acerca do construtivismo de Piaget. Ambos concordam que a criança é um ser pensante e capaz de elaborar suas próprias estruturas cognitivas, mesmo que não intencionalmente. No entanto, Papert se interrogou sobre como criar condições para que o aluno pudesse adquirir conhecimento.

Papert parte da premissa que "o ato de educar consiste em criar situações para que os aprendizes se engajem em atividades que alimentem este processo construtivo" (MALTEMPI, 2005, p. 3). O construcionismo de Papert adota uma estratégia que visa estimular o pensamento de forma criativa, possibilitando aprender através da curiosidade e envolvimento na atividade em que está realizando.

Dentre os referenciais de desenvolvimento elaborados por Piaget, Papert destaca principalmente as operações concretas. Segundo ele a construção do conhecimento deve ser "fortemente solidificada, desenvolvendo-se as entidades mentais relevantes, ampliando-se a capacidade do sujeito de operar no mundo, e uma das formas de fazer isso é experimentando e descobrindo seus caminhos" (MARTINS, 2012, p.20).

$\mathrm{Na}$ perspectiva de Papert, o estudante torna-se o foco do processo de ensino e construtor de sua aprendizagem, e o professor um orientador criativo deste conhecimento. Papert apresenta a tecnologia como um suporte para o fenômeno da aprendizagem; além disso, como um meio capaz de preparar o aluno para que exerça seu papel na sociedade. Para que isto ocorra, a escola deve se alinhar a uma forma de intervir e estimular em cada aluno a busca pelo conhecimento. Embora esse ponto possa ser quase um discurso padrão e aparente consenso em muitos autores, na prática os métodos de trabalho empregados em sala de aula podem deixar a desejar. Nunca é demais cuidar desse ponto, pois a ênfase na "educação deixa de ser a memorização da informação transmitida pelo professor e passa a ser a construção do conhecimento realizada pelo aluno de maneira significativa" (MARTINS, 2012, p. 21).

A partir da compreensão da teoria elaborada por Papert, entende-se que a escola compatível com tais fundamentos deva pensar no aluno como centro do processo de ensino. E isso só poderá ser verdade se a introdução do computador em sala de aula não acontecer como um argumento de marketing. Como se verá nesse trabalho, sequer o computador é necessário se as atividades, embora extraídas do campo da informática, tiverem como alvo trabalhar conceitos e habilidades bem definidos com os alunos.

\section{RESOLUÇÃO DE PROBLEMAS}

Como já citado, a baixa habilidade no processo de resolução de problemas pelos estudantes tem sido verificada em diferentes níveis de ensino. Para um aprimoramento do raciocínio lógico faz-se necessário estimular cedo os alunos a pensar sobre determinadas situações. Isso inclui elementos como buscar caminhos que favorecem testes através de desafios, problemas interessantes que possam ser explorados e não apenas resolvidos com operações padronizadas e utilizadas frequentemente.

O matemático Polya apresenta em sua obra consagrada, A Arte de Resolver Problemas (1995), quatro passos para facilitar a resolução de uma dada questão, que são (I) compreender o problema; (II) estabelecer um plano; (III) executar o plano e (IV) retrospecto. 
Polya detalha essas etapas, apresentando sugestões através de uma lista de itens de como o trabalho poderá ser realizado em sala de aula. Como fizeram também Piaget e Papert, ele também destacou a importância da autonomia dada ao aluno e refletiu sobre o papel do professor enquanto mediador para o sucesso da aprendizagem. "O professor que deseja desenvolver nos estudantes a capacidade de resolver problemas deve incutir nas suas mentes algum interesse por problemas e proporcionar-lhes muitas oportunidades de imitar e de praticar". (POLYA, 1995, p.3).

Compreende-se a partir dos estudos de Piaget, Papert e Polya a importância dada a autonomia do aluno para que de fato ocorra seu desenvolvimento cognitivo. Destaca-se assim, a necessidade de usar atividades e materiais que favoreçam o interesse e motivem o estudante a buscar estratégias e soluções que contribuam no processo de aprimoramento do raciocínio lógico.

\title{
O PENSAMENTO COMPUTACIONAL NO ENSINO FUNDAMENTAL
}

O pensamento computacional é chamado hoje de habilidade do século 21 . No cenário educacional, o tema vem ganhando espaço, considerando os benefícios desenvolvidos no raciocínio dos estudantes quando aplicado.

A pesquisadora Jeannette Marie Wing da Universidade de Colúmbia (USA) é considerada uma das principais promotoras do pensamento computacional. Wing (2006) destaca a importância de ensinar essa habilidade nas escolas e não apenas apresentá-la no nível superior. Mais uma vez, constata-se que independente da carreira escolhida pelo estudante, o conhecimento e as habilidades em torno da computação serão úteis na formação como um todo.

\begin{abstract}
A razão para isso é o simples fato de que a próxima geração de alunos, independentemente de qual carreira eles escolherem, já estão em um mundo com cada vez mais tecnologia, cheio de computadores e soluções baseadas na computação. Resolução de problemas lógicos, compreensão de como manipular resolução de problemas algorítmicos e abstração, juntamente com outras habilidades de pensamento computacional, são agora exigidas nos níveis primários e secundários. (MOHAGHEGH; MCCAULEY, 2016, p.1528, tradução nossa).
\end{abstract}

O pensamento computacional visa desenvolver o raciocínio lógico, a medida que fornece subsídios para resolver um problema, dividindo-o em subproblemas, que tendem a facilitar e inovar em sua resolução. Grover (2013) apud Mohaghegh e McCauley (2016, p. 1524) complementa o pensamento, pois sugere que "resolver um problema computacional envolve abordagens de pensamento lógico e algorítmico. A habilidade chave consiste em decompor logicamente um problema e criar sistematicamente um algoritmo adequado para solucioná-lo".

O pensamento computacional é visto como uma habilidade vital para hoje e para o futuro, e a sua importância equivale à de leitura, escrita, e aritmética básica (WING, 2014). Encarado assim, torna-se clara a utilidade de introduzi-lo como conhecimento curricular.

\section{METODOLOGIA}

A pesquisa foi aplicada em uma turma dos anos iniciais do ensino fundamental de uma escola pública municipal da cidade de Ponta Grossa, no estado do Paraná. Optou-se em aplicar o projeto na turma de $3^{\circ}$ ano na qual a pesquisadora era professora regente no ano de 2015. No ano seguinte, 2016, com os alunos matriculados no $4^{\circ}$ ano 
do ensino fundamental, foi dada continuidade às atividades referentes ao projeto. As aulas do projeto foram realizadas em turno regular.

As aulas foram planejadas pela pesquisadora, considerando as crianças na faixa etária de 8 a 10 anos. A partir das observações realizadas e das dificuldades evidenciadas em sala de aula, buscou-se atividades que despertassem o interesse dos alunos e que pudessem contribuir com o desenvolvimento do raciocínio lógico. Optouse por introduzir os conceitos da Ciência da Computação, baseando-se no livro Computer Science Unplugged (Bell et al 2015). Esse material é gratuito e está disponível digitalmente e em vários idiomas para download.

As atividades selecionadas e adaptadas para a pesquisa permitiram a aprendizagem de conceitos ligados ao pensamento computacional de forma lúdica, condizente com a idade dos alunos participantes da pesquisa. Foram fundamentadas numa abordagem construtivista, enfatizando a criação de novos conhecimentos e maneiras de pensar.

Sete eixos foram definidos para estruturar o trabalho: conversão de base numérica; representação de imagem; questionamento sistêmico; pensamento algorítmico; interpretação e execução de algoritmos; abstração de problemas e solução de problemas.

\section{TEMAS DESENVOLVIDOS}

Considerando as atividades realizadas, os sete temas elencados para aplicação na pesquisa junto ao grupo de alunos participantes são descritos brevemente a seguir.

Tema I - Números binários: Neste tema foram realizadas um total de três atividades. A conversão para esta base numérica foi possível a partir da utilização de algumas cartinhas confeccionadas e disponíveis no Livro Computer Science Unplugged (2011). $\mathrm{Na}$ primeira atividade, os alunos deveriam decifrar uma mensagem a partir do uso do código. As atividades seguintes tinham por objetivo verificar a apropriação do conceito, a partir das conversões realizadas tanto para base binária quanto decimal. A apresentação deste conceito para as crianças permitiu que elas pudessem compreender como o computador armazena as informações (palavras, números, imagens) a partir da conversão binária.

Tema II - Representação de uma imagem através pixels: As atividades desenvolvidas acerca do tema pixels tinham por objetivo mostrar aos alunos como o computador realiza o armazenamento de desenhos, fotografias e outras imagens utilizando apenas números.

O conceito de pixels foi um tema no qual todos os alunos apresentaram facilidade. Inicialmente realizamos coletivamente a conversão de uma imagem. A realização deste tipo de atividade requer muita atenção do estudante, visto que a composição da imagem é realizada através de sequências de números na horizontal. $\mathrm{O}$ primeiro número sempre se refere à quantidade de pixels brancos. No entanto, se iniciada pelo número 0 (zero), o pixel começará por preto.

Após a compreensão do conceito de pixel em imagens preto e branco, os alunos foram desafiados a criar imagens coloridas. Em seguida, a turma elaborou um código para cada cor, por exemplo, para formar o código da cor amarela era apresentado primeiro o número de elementos a serem pintados seguidos da cor entre parentêses $\mathrm{n}^{\mathrm{o}}$ (A). O resultado deste conceito foi muito satisfatório.

Tema III - Teoria da Informação: Para introduzir o tema da teoria da informação com os alunos, optou-se por utilizar o dicionário para exemplificar o conceito. Os alunos deveriam encontrar inicialmente a definição da palavra 'casa' em seus 
dicionários, abrindo na página que acreditavam que estaria mais próxima da palavra procurada. A partir da página aberta, as crianças deveriam avaliar se a informação estava próxima ou distante da palavra a qual estavam procurando. Esta atividade permite identificar quantas tentativas são realizadas até que o a criança consiga solucionar a atividade.

Em processamento de sinais, a quantidade de informação contida nas mensagens é mensurada pela dificuldade em adivinhá-la. Para compreensão deste conceito, foram realizadas duas atividades com as crianças. A primeira chama-se jogo das 20 perguntas, na qual primeiramente escolhe-se uma criança, que deverá pensar em um número entre 0 a 100 . As outras podem fazer perguntas à criança escolhida, que responde somente sim ou não até que se advinhe a resposta. Qualquer pergunta pode ser feita, contanto que a resposta seja especificamente 'sim' ou 'não'.

Foi apresentanto também o conceito de árvore, bastante usado em Ciência da Computação para estruturar dados de forma a permitir a busca de informações de maneira mais eficiente. A aprendizagem deste conceito tornou-se importante para que as crianças pudessem compreender a importância da síntese de ideias e a funcionalidade deste recurso nos computadores, visto que alguns sistemas podem reduzir um arquivo texto em até um quarto do seu tamanho original, proporcionando uma grande economia de espaço no armazenamento dos dados.

Tema IV - Interpretação e execução de algoritmos: O objetivo desta aula foi apresentar aos alunos métodos utilizados pelo computador para ordenar elementos de maneira rápida e eficiente. As crianças a partir dos métodos de seleção e Quicksort entenderam que existem diferentes métodos, e que cada algoritmo é desenvolvido para determinado propósito, como por exempo ordenar uma lista de nomes que tem os seus registros cadastrados aleatoriamente colocando-os em ordem alfabética.

Tema V e VI - Raciocínio lógico: Quanto ao conteúdo de situações problemas e ao tema de lógica, os mesmos não serão descritos, visto que foram desenvolvidas ao longo do ano letivo de 2015 e 2016 com a turma participante da pesquisa.

Considera-se importante que se desenvolva com os alunos o hábito de exercitar atividades desafiadoras, por meio de situações problema, desafios, jogos e enigmas que favoreçam a sua aprendizagem de diferentes formas e seja possível verificar o conhecimento de conceitos que sejam de domínio do aprendiz.

Para realização destas atividades é importante não se deter somente nos procedimentos padronizados, deixando que a criança explore os dados e busque encontrar soluções da forma que acredite ser mais condizentes.

\section{CRITÉRIOS DE AVALIAÇÃO E RESULTADOS}

Como critério de avaliação, utilizaram-se os padrões definidos por ISTE (2016), com ênfase na competência de pensar computacionalmente. Essa habilidade permite aos alunos desenvolver e empregar estratégias para resolução e compreensão de problemas, utilizando a tecnologia para verificar e testar soluções. Além disso, o processo de definição dos critérios de avaliação deste trabalho também fundamentou-se nos parâmetros da pesquisa de Lee et al (2014).

A avaliação do trabalho foi realizada por meio de uma análise pautada na associação dos conteúdos elencados nos setes eixos de conteúdos com os critérios de avaliação resumidos na segunda coluna do Quadro 1. Isso foi feito de maneira a permitir observar a evolução individual e coletiva dos alunos participantes, bem como analisar as contribuições do pensamento computacional no desenvolvimento do raciocínio lógico. 
Quadro 1 - Critérios de avaliação e resultados

\begin{tabular}{|c|c|c|}
\hline CONCEITO & CRITÉRIOS DE AVALIAÇÃO & AVALIAÇÃO DOS RESULTADOS \\
\hline $\begin{array}{l}\text { Conversão de } \\
\text { bases numéricas }\end{array}$ & $\begin{array}{l}\text { O aluno compreende que existem } \\
\text { diferentes bases numéricas, sendo a } \\
\text { decimal a convencional. } \\
\text { Ele aprende que a base binária é } \\
\text { formada por apenas dois algarismos: } 0 \\
\text { (zero) e } 1 \text { (um), e qualquer número } \\
\text { natural pode ser representado pelo } \\
\text { sistema binário. Ex: "O número } 11 \\
\text { também pode ser representado na base } \\
\text { binária como 01011". }\end{array}$ & $\begin{array}{l}\text { Observa-se que neste item avaliado, } 100 \% \\
\text { dos alunos conseguiram compreender a } \\
\text { existência de diferentes bases númericas. } \\
\text { No processo de conversão para base } \\
\text { binária compreende-se que } 96 \% \text { das } \\
\text { crianças conseguiram realizar a } \\
\text { representação a partir de sua } \\
\text { aprendizagem. }\end{array}$ \\
\hline $\begin{array}{l}\text { Representação de } \\
\text { uma imagem } \\
\text { através pixels }\end{array}$ & $\begin{array}{l}\text { O aluno compreende que imagens } \\
\text { podem ser representadas por números, } \\
\text { os quais definem a sequência dos } \\
\text { pixels. Ex: "Consigo representar uma } \\
\text { imagem usando os números } 0 \text { e 1." }\end{array}$ & $\begin{array}{l}\text { Todos os alunos que realizaram } \\
\text { atividade dominaram o processo } \\
\text { conversão de imagens, a partir } \\
\text { da } \\
\text { interpretação da sequência de pixels. }\end{array}$ \\
\hline $\begin{array}{l}\text { Questionamento } \\
\text { sistêmico }\end{array}$ & $\begin{array}{l}\text { O aluno compreende que é possível } \\
\text { definir regras que melhorem a } \\
\text { eficiência de seus questionamentos e } \\
\text { proporcionem melhores resultados. } \\
\text { Ex:"Fazendo as perguntas seguindo } \\
\text { uma lógica, posso conseguir o } \\
\text { resultado de forma mais rápida." }\end{array}$ & $\begin{array}{l}\text { Na primeira atividade proposta, verificou- } \\
\text { se que apenas 23\% dos alunos } \\
\text { conseguiram atingir a proposta da } \\
\text { atividade individuamente. No segundo } \\
\text { exercício a partir do trabalho coletivo e } \\
\text { discussões de ideias para concluir a } \\
\text { decisão, avalia-se que } 100 \% \text { atingiram } \\
\text { êxito na atividade. }\end{array}$ \\
\hline $\begin{array}{l}\text { Pensamento } \\
\text { algorítmico }\end{array}$ & $\begin{array}{l}\text { O aluno organiza o seu pensamento de } \\
\text { forma a fornecer subsídios para } \\
\text { resolver problemas, podendo estes } \\
\text { serem subdivididos em subproblemas, } \\
\text { que tendem a facilitar o seu processo } \\
\text { de resolução. Ex: "Consigo encontrar } \\
\text { qualquer palavra em um dicionário se } \\
\text { seguir um passo a passo e repeti-lo até } \\
\text { encontrar a palavra". }\end{array}$ & $\begin{array}{l}\text { No processo de transcrição do algoritmo } \\
\text { da dobradura, apenas } 17 \% \text { das crianças } \\
\text { conseguiram realizar a atividade proposta. } \\
\text { Apesar disso, os alunos demonstram a } \\
\text { aprendizagem do conceito quando foram } \\
\text { questionados e reportaram que no } \\
\text { computador há a necessidade de se passar } \\
\text { informações precisas para que ocorra a } \\
\text { interpretação correta, visto que, } \\
\text { diferentemente dos seres humanos, a } \\
\text { máquina so irá realizar as instruções } \\
\text { repassadas literalmente. }\end{array}$ \\
\hline $\begin{array}{lr}\text { Interpretação } & \mathrm{e} \\
\text { execução } & \mathrm{de} \\
\text { algoritmos } & \end{array}$ & $\begin{array}{lcrr}\text { Possibilita ao } & \text { aluno interpretar } \\
\text { instruções de } & \text { forma } & \text { correta, } \\
\text { executando-as de } & \text { maneira a } & \text { alcançar } \\
\text { uma solução. Ex: "Se eu não seguir as } \\
\text { instruções corretamente, o resultado } \\
\text { ficará diferente do esperado". }\end{array}$ & $\begin{array}{l}\text { Optou-se por desenvolver as atividades } \\
\text { coletivamente, obtendo assim um } \\
\text { resultado de } 100 \% \text { de aproveitamento nos } \\
\text { grupos, verificado a partir das trocas de } \\
\text { ideias e argumentos utilizados pelas } \\
\text { crianças na compreensão do algoritmo. }\end{array}$ \\
\hline $\begin{array}{l}\text { Abstração } \\
\text { problemas }\end{array}$ & $\begin{array}{l}\text { O aluno consegue identificar a origem } \\
\text { do problema, extraindo as informações } \\
\text { relevantes para a solução do mesmo. } \\
\text { Ex: "Eu entendi o que devo fazer para } \\
\text { resolver, e preciso calcular com esses } \\
\text { números". }\end{array}$ & $\begin{array}{l}\text { Neste processo, verificou-se a partir do } \\
\text { acompanhamento contínuo e nas situações } \\
\text { problemas propostas que } 81 \% \text { conseguiam } \\
\text { abstrair as informações para buscar uma } \\
\text { possível solução. }\end{array}$ \\
\hline $\begin{array}{l}\text { Solução } \\
\text { problemas }\end{array}$ & $\begin{array}{l}\text { O aluno organiza as informações e } \\
\text { analisa as variáveis, formando uma } \\
\text { solução. Ex: "A partir destas } \\
\text { quantidades, consigo resolver de duas } \\
\text { formas, e ter o mesmo resultado". }\end{array}$ & $\begin{array}{l}\text { A partir do acompanhamento das } \\
\text { atividades verificou-se que cerca de } 70 \% \\
\text { dos alunos conseguiam encontrar as } \\
\text { soluções propostas, sejam através do uso } \\
\text { padronizado de operações ou de acordo } \\
\text { com seu raciocínio lógico. }\end{array}$ \\
\hline
\end{tabular}

Fonte: Adaptado de Denner, Werner e Ortiz (2011), Lee et al (2014) e ISTE (2016)

Na terceira coluna do Quadro 1 apresenta-se uma análise global sobre os resultados obtidos acerca dos critérios de avaliação definidos, baseando-se em atividades aplicadas e observações realizadas pela pesquisadora. $\mathrm{O}$ interesse primordial 
dessa análise é pautar o trabalho de outros pesquisadores, em relação a pontos que mereçam mais cuidado de planejamento. Destaque-se outra vez que as atividades em si, realizadas em sala de aula, devem ser definidas caso a caso, segundo o contexto escola, sala de aula, alunos.

A partir das atividades desenvolvidas com o grupo de alunos percebe-se que a aprendizagem dos conceitos ligados a Ciência da Computação se deu de forma satisfatória. Proporcionar às crianças o conhecimento a temas ligados á área da Ciência da Computação desde os primeiros anos de escolarização tornam-se fundamentais na atual sociedade, considerando que a tecnologia avança e computadores e soluções computacionais estão envolvidos cada vez mais em nossas vidas diárias. Portanto, todos os níveis de educação devem em algum momento propiciar que crianças e jovens se preparem para um mundo cada vez mais digital.

Desta forma, conclui-se que a aprendizagem de conceitos computacionais da forma apresentada atrai a curiosidade das crianças, de modo a estimular que encontrem soluções rápidas para diferentes problemas. A proposta desta pesquisa permitiu que além da aprendizagem de conceitos ligados a Ciência da Computação, as crianças compreendessem a possibilibildade de desenvolver mecanismos de interações e descobertas através da máquina, permitindo que eles possam atuar sobre o computador e não somente utilizar os recursos previamente disponíveis no equipamento.

\section{CONSIDERAÇÕES FINAIS}

Através do presente trabalho, foi possível observar que com a introdução de conceitos da Ciência da Computação logo nos anos iniciais de escolarização, os estudantes desenvolveram habilidades que vão além de pensar logicamente, como a autonomia e a capacidade de transformar um problema geral em parcelas menores para sua resolução. Além disso, os participantes adquiriram conhecimentos antes delimitados a áreas específicas, como computação e engenharias.

Compreende-se também, que para maiores contribuições no desenvolvimento do raciocínio lógico dos alunos, faz-se necessário dar continuidade às atividades aplicadas, de modo a nivelar o grau de complexidade a ser aprendido.

$\mathrm{O}$ estímulo ao raciocínio lógico e as habilidades ligadas ao pensamento computacional podem ser especialmente importantes no atual contexto, onde a tecnologia traz inovações constantemente e a sociedade requer novas competências. Exige-se cada vez mais do estudante capacidades como pensamento sistêmico, autonomia, comunicação, relacionamento interpessoal, eficácia em processos e soluções de problemas.

Portanto, o objetivo apresentado na pesquisa foi respondido de forma satisfatória, visto que, a partir das atividades desenvolvidas acerca do pensamento computacional com o grupo de alunos participantes, foi possível observar a assimilação de novos conceitos ligados à Ciência da Computação, visto que os alunos passaram a dominar os principais termos trabalhados, os quais foram abordados no projeto; além disso, apresentaram uma maior maturidade na resolução dos problemas, mostrando que já conseguem refletir de maneira mais criteriosa visando encontrar soluções para as atividades propostas.

\section{Referências bibliográficas}

BELL, T.; WHITTEN, I.; FELLOWS, M. Computer Science Unplugged (Ensinando

Ciência da Computação sem o uso do computador) Universidade de Canterbury, Nova Zelândia, 2011. 
EVANS, Jonathan St BT. In two minds: dual-process accounts of reasoning. Trends in cognitive sciences, v. 7, n. 10, p. 454-459, 2003.

ISTE. International Society for Technology in Education. Disponível em:< www.iste.org/standards/standards/for-students-2016:. Acesso 01 set. 2016.

KRAMER, Jeff. Is abstraction the key to computing? Communications of the ACM, v. 50, n. 4, p. 36-42, 2007.

LEE, T. Y.; MAURIELLO, M. Louis; AHN, J; BEDERSON, B.B. CTArcadeD: Computational thinking with games in school age children. International Journal of Child-Computer Interaction, volume 2, p. 26-33, jan.2014. Disponível em < http://www.sciencedirect.com/science/article/pii/S2212868914000208 >. Acesso em: 16 jul. 2016.

PIAGET, Jean. A epistemologia genética e a pesquisa psicológica. Rio de Janeiro: Freitas Bastos, 1974.

MALTEMPI, M. Vinicius. Novas Tecnologias e Construção de Conhecimento: Reflexões e Perspectivas. In: V Congresso Ibero-americano de Educação Matemática (CIBEM). Porto, Portugal, 2005. Disponível em < http://www.rc.unesp.br/igce/demac/maltempi/Publicacao/Maltempi-cibem.pdf $>$. Acesso em: 09 jul. 2016.

MARTINS, Amilton R. de Quadros. Usando o Scratch para potencializar o pensamento criativo de crianças do ensino fundamental. Dissertação (Mestrado em Educação) - Programa de Pós- Graduação em Educação, Universidade de Passo Fundo, Passo Fundo, 2012. Disponível em http://www.upf.br/ppgedu/images/stories/defesadissertacao-amilton-rodrigo-de-quadros-martins.PDF. Acesso em: 15 abr. 2015.

MOHAGHEGH, Mahsa; MCCAULEY, Michael. Computational Thinking: The Skill Set of the 21st Century. International Journal of Computer Science and Information Technologies, Vol. 7, 2016, p. 1524-1530. Disponível em: < http://www.ijcsit.com/docs/Volume\%207/vol7issue3/ijcsit20160703104.pdf >. Acesso em 24 jul.2016.

POLYA, G. A arte de resolver problemas. Rio de Janeiro: Editora Interciência, 1995.

Tim Bell, Ian H. Witten and Mike Fellows. CS Unplugged. Publicado por "Lulu.com". Versão digital disponível em http://csunplugged.org/books>. Acesso: 17 out. 2017.

WING, Jeannette M. Computational Thinking. 2006. Disponível em:< https://www.cs.cmu.edu/ 15110-s13/Wing06-ct.pdf> Acesso em 18 jul.2016.

WING, Jeannette M. Computational Thinking Benefits Society. 2010. Disponível em: <http://socialissues.cs.toronto.edu/index.html\%3Fp=279.html>.Acesso em 18 jul.2016. 